\title{
Optimization of cold and heat storage volumes of a thermal-energetic system with IDA ICE
}

\author{
Marco Borer, Moritz Zwahlen, Manuel Frey \\ Gruner Roschi AG, Bauklimatik \& Simulationen, Köniz, Schweiz
}

\begin{abstract}
Using a practical example, the heat and cold storage volume is analyzed and optimized using the tools integrated in IDA ICE. In practice, the simulation models must be partially simplified for efficiency reasons. The level of detail of the storage tanks should be chosen in such a way that the temperature behavior of the simplification corresponds as closely as possible to the behavior of a non-simplified model. Hence, the required findings can be obtained from the simulation and optimizations can be carried out efficiently. The investigation showed that certain model simplifications are legitimate and that the optimization tools in IDA-ICE can be used reasonably for different tasks of an overall optimization.
\end{abstract}

\section{Introduction}

Gruner Roschi AG has the HVAC, electrical and plumbing planning mandate for a total renovation of two large service buildings with a net floor area of $40,000 \mathrm{~m}^{2}$ in Zurich. The retrofitting will begin in summer 2019. Based on a detailed system energy simulation in IDA ICE, the heating and cooling systems were designed, and the district heating connection and cooling machine dimensioned. Based on this useful energy simulation, a model of the thermal-energetic system was constructed. All important components were mapped in IDA ICE to analyze their operation and interrelations in detail. The main components of the system are the chiller, the free cooling system, the recooling system, the district heating connection and the heat and cold storage tanks. Added to this are all consumers such as domestic hot water, air heaters and coolers as well as room heating and cooling. In the simulation, only two storage tanks with an adjusted volume are simulated. However, in reality nine heat storage tanks and twelve cold storage tanks with a capacity of 5 cubic meters each are used in series production. Figure 1 shows a general overview.

Even today, although computer-aided dimensioning has long been possible, the design of thermal-energetic plants is still largely based on empirical values and static calculation methods. Simulations in general offer many advantages that previous working methods cannot. The dynamics of the elements within the system, their interplay and the processes acting on the system are ideally determined by a simulation. This enables the

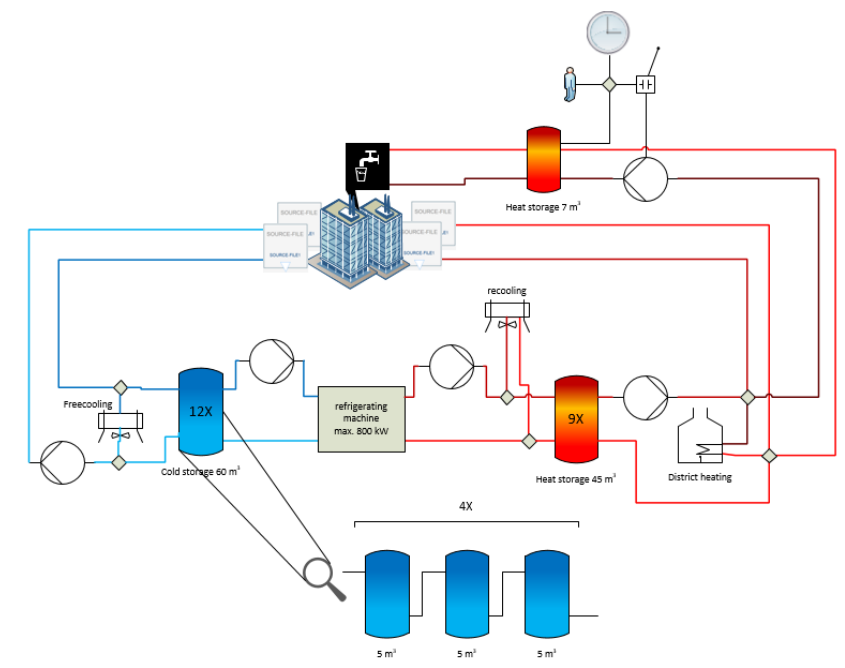

Figure 1: Overall view of the thermal-energetic system to be optimized

simulation engineer to show the planner which dynamics occur in the system and if necessary how to deal with them. The larger knowledge of a thermal-energetic system, which is generated through the simulation, can be used to optimally dimension system components, to test the effectiveness of innovative ideas or to determine the limits of the system. Optimizing thermal-energetic systems in buildings, contributes to more energy-efficient buildings by identifying and taking potential improvements into account as early as the planning phase.

The aim of this optimization is to optimally distribute the storage volume of $105 \mathrm{~m}^{3}$ to the heat and cold storage to reduce the electrical energy required for the chiller as much as possible. Important information on the procedure and theoretical principles are described in the next chapter.

\section{Methods}

In this chapter, information on the IDA ICE software used and the procedure for optimizing the storage volume is explained.

\section{IDA ICE}

IDA ICE is a simulation program of the Swedish software manufacturer EQUA, which has been developing simulation software for buildings, tunnels and energy 
systems since the early 1990s. IDA ICE is an innovative, detailed, dynamic and validated simulation program for the assessment of indoor climate and energy consumption of entire buildings. The physical models are based on the latest scientific knowledge and thus generate the best available comparison of model and reality. The calculation results are convincing due to their high conformity with measurement data. Extensions for the country-specific needs of users, such as climate data, standard extensions and translation into various languages, make the simulation software a widely used tool worldwide (Equa, 2018). There are more than 2000 active IDA ICE users worldwide. (Ruepp, 2018)

At Gruner Roschi AG, IDA ICE is mainly used because of the flexible and efficient application possibilities of building and system simulations. In addition, the simulation program convinces with its very transparent result evaluation and the good traceability of the calculations. Another advantage, especially for building simulation, is the possibility to import geometric information from 3D models (e.g. IFC file).

Since IDA ICE version 4.8 four different analysis and optimization tools are available: Parametric runs, GenOpt, Sensitivity Analysis and Monte-Carlo. Parametric runs can be used to conduct parameter studies and GenOpt to optimize the system with selected parameters to specific targets. Sensitivity analysis can be used to investigate extreme values of selected parameters. With Monte-Carlo, the selected parameters can be randomly varied within the specified range. Parametric runs, GenOpt and Monte-Carlo together with multiple linear regression analysis are used to optimize the storage volume.

\section{Optimization with IDA ICE and GenOpt}

IDA ICE can communicate with the GenOpt optimization software via text files. Various optimization algorithms can be used in GenOpt. Two suitable algorithms for optimizing problems in IDA ICE are Particle Swarm Optimization and Generalized Pattern Search (GPS). The combination of these well-known optimization algorithms enables reliable and fast optimization results to be used cost-efficiently even in the conflicting areas of a planning and engineering office. The use of algorithms for the automatic optimization of plant systems as an integrated step in a planning process with application in a HVAC planning and engineering office has hardly been used so far. The goal of this approach is, besides optimizing the system, also to detect possible problems, which could occur during operation, even before the start of construction. Finding the optimum for the investigated system required between 250 and 300 simulations in IDA ICE, which with the investigated plant model and existing hardware, corresponds to a simulation time of approximately 24 hours. For use in an engineering office, faster optimization is desirable. However, for this study, the duration of the optimization was sufficiently short and was not further improved. In the future, the optimization time could be shortened by using other algorithms or more powerful computers. The extent to which the number of parameters used for optimization influences the optimization duration was not considered.

\section{Procedure}

For optimization, Parametric runs and GenOpt are used to find the best parameter values for the system. To optimize the storage volumes in a short simulation time, various simplifications are made. These are explained in more detail in the chapter "Models in IDA ICE" and the differences to the models without simplifications are shown in "Results".

\section{Models in IDA ICE}

Two simplifications are examined. The aim of these investigations is to find out which simplifications can be made in a simulation model to still obtain realistic results. The simplifications are examined in two steps to gain comparable results. The simplifications are also illustrated graphically in Figure 2.

\section{Simplification: connection type}

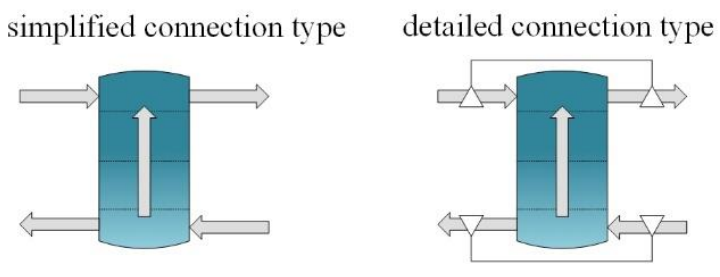

\section{Simplification: number of storage tanks}

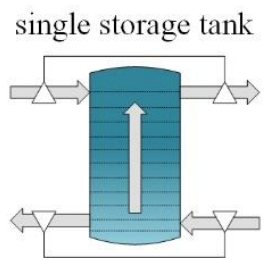
many storage tanks

Figure 2: Two simplifications were investigated

The system has a cold and heat storage volume, both of which are connected to the system parallel to the discharge. In reality, the total volume of these two storages consists of several individual tanks through which the water flows in series. The cold storage volume consists of 12 and the heat storage volume of 9 storage tanks with a volume of 5000 liters each. In a first step, the influence of the connection type is investigated using the example of the cold storage tank. To reduce the modelling effort, the model should be as simple as possible, but nevertheless represent the real process. Two IDA ICE models were created to investigate the simplifications explained in the following.

In the real system, the storage volumes have a bypass. Therefore, only the excess amount of water from the chiller that is not needed by the user, flows into the storage tank. The inflow and outflow of the storage tanks varies 
depending on the charge and discharge. In the simplified model, this bypass is neglected and only one storage tank is simulated. The flow of the chiller is connected directly to the lowest layer of the storage tank and the return directly to the uppermost storage layer, so the entire mass flow must stream through at least one storage layer. This simplification means that more water flows into and out of the bottom and top storage layers compared to the realistic connection. The investigation will show whether the simplified model conducts more or less energy to further storage layers than the detailed model. The focus of this investigation lies in the comparison of the energy flows, additionally the temperatures are compared. The second investigation aims to find out whether it is legitimate to represent a series circuit with only one single storage. The total storage volumes are connected to the system with a bypass as in the previous study and thus build on the previous study. The simplified model has only one tank, in which each storage layer represents a single storage tank in the series connection. It is to be shown whether the tanks in series behave differently than the layers of a single storage tank. The focus of this investigation lies on the storage temperatures. In addition, the energy flows into and out of the storage tank are also compared.

\section{Results}

This chapter summarizes the influences of the simplifications and the optimization results.

\section{Storage connection}

The effect of the connection type on the heat and cold storage is examined in more detail here using the example of cold storage. Figure 3 shows the mass flow into the storage tank in the model with the bypass as planned for the real system. Figure 4 shows the mass flow into the storage tank with the simplified piping without bypass.

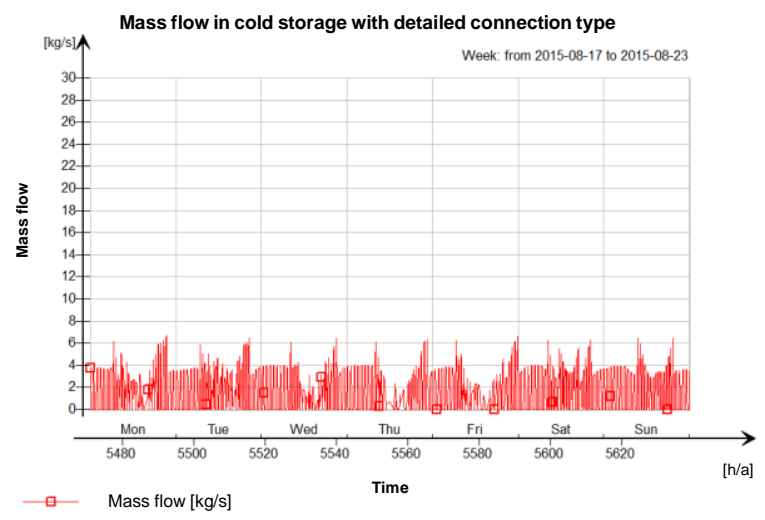

Figure 3: Mass flow in cold storage with detailed connection type

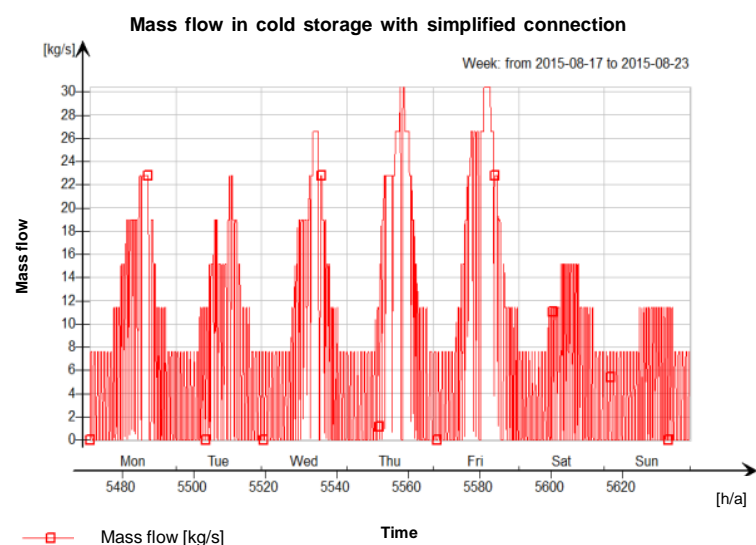

Figure 4: Mass flow in cold storage with simplified connection type

The mass flows in and out of the cold storage differ from each other due to the different connection types. In the simplified model, where the mass flow of the water always flows through the cold storage tank, the mass flow is up to four times greater than in the model with the detailed connection type. Table 1 shows the results of the simulations. The figures clearly show how the simplification of the storage connection affects the energy flows into and out of the cold storage.

Table 1: Energy flows of the cold storage tank with simplified connection compared to the detailed type of connection

\begin{tabular}{|l|l|l|}
\hline $\begin{array}{l}\text { Heat flow in cold } \\
\text { storage }\end{array}$ & $\begin{array}{l}\text { Heat flow out cold } \\
\text { storage }\end{array}$ & $\begin{array}{l}\text { Transmission heat } \\
\text { flow through cold } \\
\text { storage }\end{array}$ \\
\hline $194 \%$ & $194 \%$ & $92 \%$ \\
\hline
\end{tabular}

Although the simplified connection means that almost twice as much cooling energy flows into and out of the cold storage, the cooling energy, which moves vertically through the storage layers, drops by $8 \%$. The type of connection also causes the storage temperatures to deviate from each other. Especially in summer, when high mass flows are needed for cooling, different layer temperatures can be observed.

\section{Series connection and number of storages}

The example of the heat storages illustrates how the series connection affects the temperature behavior in the storage tanks. In the cold storage a similar temperature behaviour can be observed, but the effect is less pronounced. Figure 5 shows the duration lines of the layer temperatures of the single storage tank (simplified model) and Figure 6 shows the duration lines of the mean storage tank temperatures of several storage tanks in series connection (detailed model). 


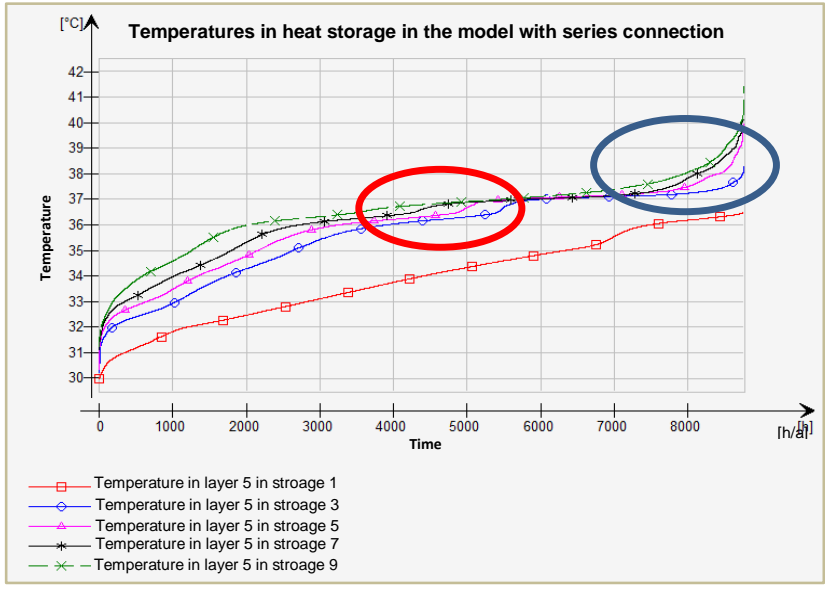

Figure 6: Temperatures in heat storage in the model with series connection (detailed model)

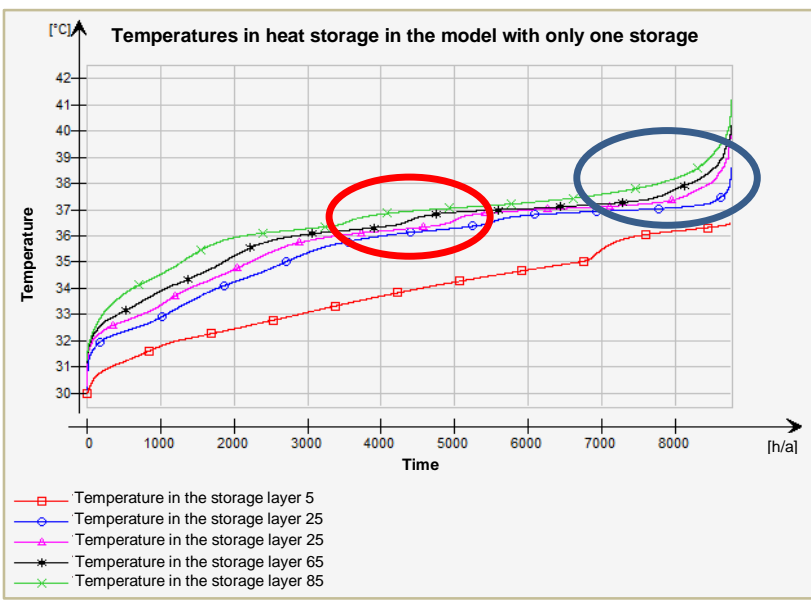

Figure 5: Temperatures in heat storage in the model with a single storage (simplified model)

The series connection only makes a small difference energetically. In the model with a single storage tank, $3.1 \%$ more heat was stored than in the comparable model with twelve storage tanks. This corresponds to an energy quantity of $4000 \mathrm{kWh}$ under the same conditions, which is only about $0.5 \%$ of the waste heat of the chiller. The temperatures in the storage tanks show a larger difference. Especially, when fewer storage layers are simulated, the layer temperatures from the simplified model diverge from those in the more detailed model. The higher the number of layers in the storages, the smaller the deviation of the duration lines become. Even with the same number of layers in the simplified model and in the detailed model, a small deviation between the two models is visible in the uppermost storage layer. See Figure 5 and 6. No differences are visible in the storage layers below.

\section{Optimization results}

The following two graphs show the influence of storage volumes on the most influenced system components and sizes in the system. The cold storage volume mainly influences the running time of the chiller in the lowest stage. The lowest stage is the most efficient and should therefore be in operation for as long as possible. With a smaller cold storage volume, the running time of the chiller in the lowest stage decreases and the average cooling capacity increases (Figure 7).

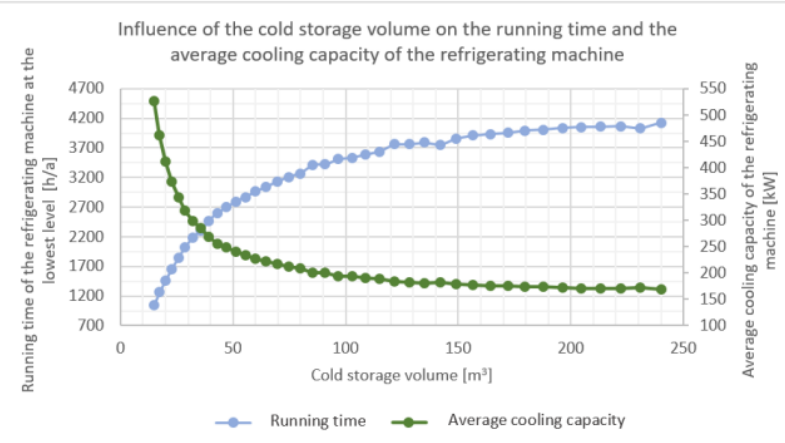

Figure 7: Influence of the cold storage volume on the running time and cooling capacity of the chiller

Compared to the cold storage volume, the volume of the heat storage tank does not have a strong influence (Figure 8 ). The most influenced energy variable is the used waste heat from the chiller. It is higher for large heat storage volumes than for small ones. Since more of the total waste heat is stored in the heat storage, less waste heat must be removed through recooling. The total storage volume of $105 \mathrm{~m}^{3}$ is optimized with GenOpt, so that the electricity requirement for the chiller is minimal. In a further optimization, recooling is minimized for comparison. The required power consumption of the chiller is used as the target function. Table 2 shows the optimum storage volumes and Table 3 the lowest values for the power requirement of the chiller. The combined storage volume of the two tanks must not exceed $105 \mathrm{~m}^{3}$.

\section{Choice of target variable}

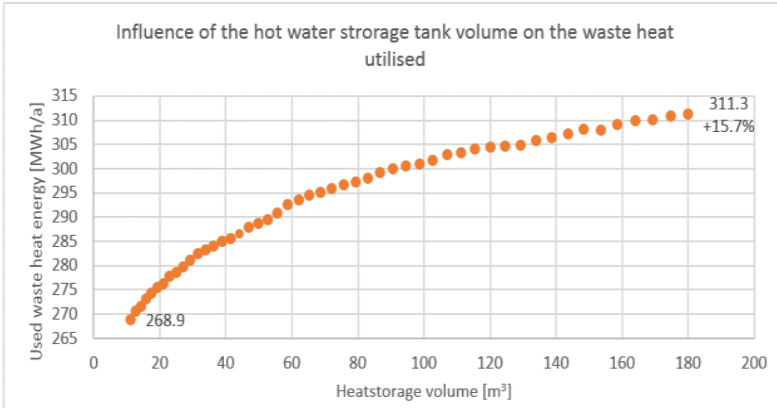

Figure 8: Influence of the hot water storage tank volume on the waste heat utilised

The choice of the target variable for an optimization of the storage volume requires the consideration of several factors. The ideal storage volume can vary depending on the selected target variable. As an example, the cooling 
energy of the chiller and the recooling energy are investigated here, since it was expected before the investigation that these two variables would be influenced by the storage volume distribution. The recooling energy proved to be unsuitable for defining the storage volume. For the minimization of the recooling energy, a larger heat storage volume was expected than that resulting from the optimization. As the investigation shows, the recooling energy is mainly dependent on the freecooling energy brought into the system, which optimally has a small cold storage volume, as it can be used more frequently due to the control system. By minimizing the recooling energy, the freecooling energy is actually maximized and the heat storage volume is designed accordingly. Overall, the minimization of the recooling energy achieved the smaller improvement of both target functions. Designing the storage volume on the basis of the cooling energy of the chiller is the better choice in this study, as an overall better result was achieved. In order to find optimum parameter values for the overall system, an existing target variable must be found in the system which meets all requirements or a superordinate target value must be generated which summarizes all relevant system criteria and, if necessary, evaluates them. For example, the total primary energy consumption could be such a target.

Table 2: Optimal storage volume

\begin{tabular}{|l|l|l|}
\hline Target size & $\begin{array}{l}\text { cold storage } \\
\text { volume }\left[\mathbf{m}^{3}\right]\end{array}$ & $\begin{array}{l}\text { heat storage } \\
\text { volume }\left[\mathbf{m}^{3}\right]\end{array}$ \\
\hline $\begin{array}{l}\text { Cooling energy } \\
\text { chiller }\end{array}$ & $74 / 105$ & $31 / 105$ \\
\hline Recooling energy & $52 / 105$ & $53 / 105$ \\
\hline
\end{tabular}

Table 3: Minimized cooling and recooling energy

\begin{tabular}{|l|l|l|}
\hline Target Variable & $\begin{array}{l}\text { Cooling } \\
\text { energy chiller } \\
{[\mathbf{k W h}]}\end{array}$ & $\begin{array}{l}\text { Recooling } \\
{[\mathbf{k W h}]}\end{array}$ \\
\hline $\begin{array}{l}\text { Cooling energy } \\
\text { chiller }\end{array}$ & $714^{\prime} 441$ & $524^{\prime} 509$ \\
\hline Recooling energy & $719^{\prime} 013$ & $525^{\prime} 927$ \\
\hline
\end{tabular}

\section{Discussion}

Under this point the results are discussed.

\section{Connection type}

Although the simplified connection type leads to almost double the amount of cooling energy passing through the lowest and uppermost layers of the cold storage, the energy flow in the intermediate layers drops by $8 \%$. How much water and thus also cold or heat is led through a storage tank depends on the model, but in reality, also, on the charge and discharge of the storage. The storage serves as a compensation tank. If the supply of the chiller is higher than needed by the user, water is forced into the storage. In the opposite case, water is discharged from the storage tank. The difference between the two models can be explained by the fact that different charging and discharging mass flows are caused by the different connection types to the system, whereby the pump controls also had to be adapted. This also changes the charging and discharging of the storage tanks. Further investigations showed that the pump controls affected the difference even more than the connection type. Since the pump control was also adapted for the investigation of the connection type, it can be assumed that the differences between the two models are due to this. To optimize the storage volume, the simplified model is further used, but the pump control is transferred to the optimization model to get closer to the principle scheme of the planner.

\section{Series connection}

A single heat storage, which represents several heat storages in series, shows differences in temperature behavior compared to the detailed model with several tanks. The more layers the individual storage tank has, the smaller are the differences. In Figure 5 and Figure 6, the simplified model with only one storage tank and the detailed connection type is compared to the model with many storage tanks in series connection. The differences in the duration lines of the storage temperatures are small. In the area circled in red, the temperature of the uppermost reference layer 85 is warmer in about $1000 \mathrm{~h} / \mathrm{a}$ than that of the underlying layers. In the detailed model with nine storage tanks, the temperatures are closer together. The second difference, encircled in blue, is similar. Again, the top layer is warmer compared to the detailed model with nine tanks. Whether these differences are still relevant, for example, for a study of the efficiency of chillers, still needs to be examined. The observed effect is that storage tanks with a larger temperature difference between the top and bottom layers have a stronger stratification. Hot water rises to the top. This causes the lines in the duration diagram to shift to the left. Due to the stronger stratification, the higher layers have higher temperatures for a longer period. In the model with nine storages, the stratification effect occurs in all storages. The temperature differences between the top and bottom layers in these tanks are smaller. As a result, the stratification in the storages is smaller and the temperatures in the tanks become more uniform compared to the simplified model. Even with the same number of layers, the effect of the stronger stratification cannot be fully compensated in the simplified model. For very sensitive investigations, it is therefore advisable to model nine storages instead of just one with the equivalent number of layers. Since no relevant differences were observed for the energetic storage volume optimization, the optimization was done with the simplified model.

\section{Discussion of the optimization results}

When dividing the total storage volume of $105 \mathrm{~m}^{3}$ into heat and cold storage tanks, a good overall result is achieved when the cold storage tank is $74 \mathrm{~m}^{3}$ and the heat storage tank is $31 \mathrm{~m}^{3}$ in size. With these storage sizes, the 
cooling energy requirement of the chiller and thus also the electricity requirement are the lowest. An additional benefit from less cooling energy from the chiller is the generation of less waste heat from the chiller meaning less recooling is required. The negative effect of the system needing more heat due to the smaller amount of waste heat being available from the chiller did not occur. This is due to the relatively small storage tanks in relation to the size of the system. The heat storage is almost always saturated. A smaller amount of waste heat therefore has a direct impact on recooling. The results of minimizing the recooling energy show that the heat storage must be larger than the cold storage to reduce recooling. Nevertheless, this optimization resulted in a smaller improvement compared to the optimization of the chiller cooling energy. The amount of recooled heat was higher when the recooling energy was used as the target variable to minimize compared to when the chiller cooling energy was used as the target variable. This is due to their correlation in the system, since the amount of recooled heat mostly depends on how much freecooling energy can be brought into the system. GenOpt chose storage tanks that allow as much free cooling as possible, but neglected the cooling energy of the chiller, which also reduces recooling. This led to an optimization of the wrong variable in the system. The choice of the best target variable for optimizing the storage volume proved to be difficult during project work. Minimizing the chiller cooling energy is better suited as a target function for this optimization. It is essential to check whether the best simulation results can be achieved with the target function used. To do this, several optimizations must be made and compared. The use of Monte-Carlo in combination with multiple linear regression analysis is well suited to check this without high simulation effort. Alternatively, a target variable can be created which contains all the values to be investigated and, if necessary, evaluates them. As a result of this investigation $74 \mathrm{~m}^{3}$ for the cold storage and $31 \mathrm{~m}^{3}$ for the heat storage are suggested as the optimal. With the proposed distribution of the storage volume, the running time of the chiller in the most efficient operating stage and therefore the chiller efficiency and recooling energy are managed best. By minimizing the cooling energy requirement, approx. 4.5 MWh less cooling energy is obtained from the chiller. The minimization of recooling resulted in a reduction of only approx. 1.5 MWh recooling energy.

\section{Conclusion}

For purely energetic optimization, the type of connection and series connection can be simplified in the model. As the simulation results show, the stored heat or cold is mainly determined by the mass flows in the system. These are influenced by the control logic of the pumps in the system and should correspond as closely as possible to the control logic of the planner for realistic simulations. The type of connection and number of storage tanks only have a minor influence on the stored heat or cold compared to the control of the pumps. The temperature in the cold storage is influenced by the connection type especially in summer, when higher mass flows stream into the storage. The temperatures in many tanks with a series connection deviate from the temperatures of a single tank representing the same storage. The more layers the single storage tank has, the closer it is to the temperature behavior of the storage tanks in series connection. Even with the same number of layers, there are still temperature differences in the upper layers as can be seen in the duration line diagram. For investigations in which the temperatures in the storage tanks are decisive, it is therefore recommended to use the detailed models. The paper showed that the representation of the simulation results and the choice of the tool for the investigation depends on the number of parameters investigated. Figure 9 shows which display and evaluation options are suitable depending on the number of parameters used.

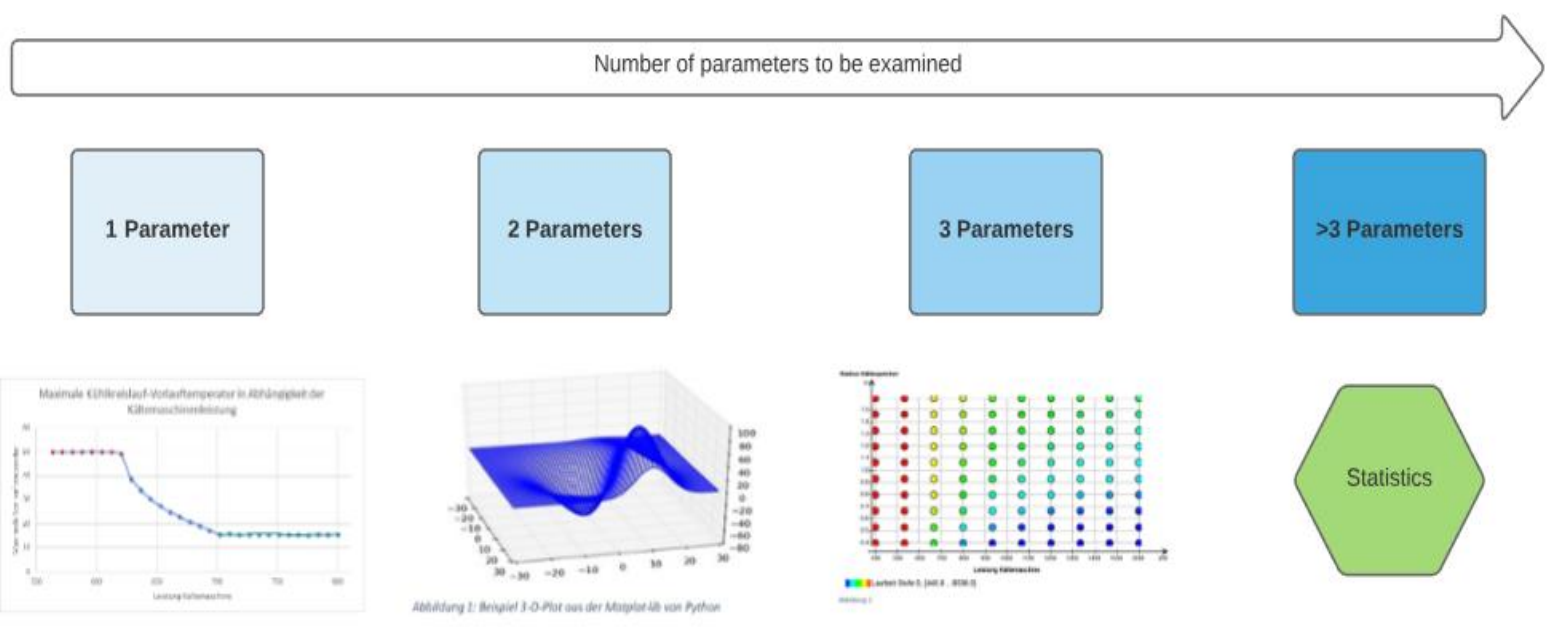

Figure 9: suitable display formats depending on the number of parameters to be examined 


\section{References}

Wetter, M., 2016. simulationresearch.

[Online] Available at: https://simulationresearch.lbl.gov/GO/jdoc/index.htm 1?genopt/algorithm/GPSPSOCCHJ.html

[Zugriff am 23.11.2018]

Ruepp, D., 2018. Dip. Ing. [Interview] (29.11.2018)

Röber, M., 2010. qucosa.

[Online] Available at: http://www.qucosa.de/fileadmin/data/qucosa/docume nts/7767/Diplomarbeit_Marcel_Roeber.pdf

[Zugriff am 26.10.2018]

Equa.se, 2018. Equa Building Performance Simulation.

[Online] Available at:

https://www.equa.se/de/about-us/building-

performance-simulation

[Zugriff am 14.09.2018]

inivie.org, 2003.

[Online] Available at:

http://www.inive.org/members_area/medias/pdf/Iniv e/IBPSA/UFSC89.pdf

[Zugriff am 26.10.2018] 